\title{
MÉTODOS DE GESTÃO DE PROJETOS E SUCESSO DOS PROJETOS: UM ESTUDO QUANTITATIVO DO RELACIONAMENTO ENTRE ESTES CONCEITOS
}

\author{
PROJECT MANAGEMENT METHODOLOGIES AND PROJECT SUCCESS: A \\ QUANTITATIVE STUDY OF THE RELATIONSHIP BETWEEN THESE CONCEPTS
}

\section{Leandro Alves Patah}

Doutor em Engenharia de Produção pela Universidade de São Paulo - USP

Professor do Programa de Mestrado Profissional em Administração - Gestão de Projetos da Universidade Nove de Julho - PMPA-GP/UNINOVE

E-mail: leandro.patah@uninove.br (Brasil)

\section{Marly Monteiro de Carvalho}

Doutora em Engenharia de Produção pela Universidade Federal de Santa Catarina - UFSC

Professora da Escola Politécnica da Universidade de São Paulo - POLI/USP

E-mail: marlymc@usp.br (Brasil) 


\title{
MÉTODOS DE GESTÃO DE PROJETOS E SUCESSO DOS PROJETOS: UM ESTUDO QUANTITATIVO DO RELACIONAMENTO ENTRE ESTES CONCEITOS
}

\begin{abstract}
RESUMO
Investigar o relacionamento entre a adoção de metodologias de gestão de projetos e o sucesso em projetos é o principal objetivo desse trabalho. Para isso a abordagem metodológica consistiu em um levantamento de campo longitudinal, conduzido em 3 países, Argentina, Brasil e Chile, por um período de 3 anos. Foram obtidos dados de 1387 projetos. Os resultados permitiram evidenciar influência positiva e significativa do grau de implementação de métodos de gestão de projetos no sucesso dos mesmos, do ponto de vista de prazo.
\end{abstract}

Palavras-chave: Gestão de Projetos; Sucesso em Projetos; Indicadores de Desempenho.

\author{
PROJECT MANAGEMENT METHODOLOGIES AND PROJECT SUCCESS: A \\ QUANTITATIVE STUDY OF THE RELATIONSHIP BETWEEN THESE CONCEPTS
}

\begin{abstract}
To investigate the relationship between the adoption of project management methodologies and the project success is the main objective of this work. The methodological approach consisted in a longitudinal field research conducted in 3 countries: Argentina, Brazil and Chile, during a period of 3 years. Data from 1387 projects were obtained. The results show a positive and significant influence from the implementation degree in the project success, from the schedule point of view.
\end{abstract}

Keywords: Project Management; Project Success; Performance Indicators. 


\section{INTRODUÇÃO}

O gerenciamento de projetos, como teoria, encontra-se bem desenvolvido e é aceito nos dias de hoje no mercado como uma competência necessária para as organizações. Vários métodos e técnicas têm sido desenvolvidos cobrindo todos os aspectos de um projeto, desde sua concepção até a entrega final dos produtos produzidos pelo mesmo. Apesar disso, a gestão de projetos permanece um desafio altamente problemático, uma vez que uma grande quantidade de projetos excede seus orçamentos, atrasam ou falham em cumprir seus objetivos, como evidenciado por diversas pesquisas (DAI; WELLS, 2004; THE STANDISH GROUP, 2009; WHITE; FORTUNE, 2002).

Nos últimos anos muitas empresas têm gasto quantidades significativas de recursos em gerenciamento de projetos. O PMI (2009) apresenta um número de US\$ 12 trilhões, um quinto do valor do PIB mundial, como o valor a ser investido em projetos em cada um dos anos da atual década.

Apesar disso, não se encontra uma grande quantidade de pesquisas a respeito do retorno do investimento em métodos de gerenciamento de projetos. Com isso, mesmo com o alto grau de investimento em projetos e o crescente número de gerentes de projetos qualificados, algumas questões sobre os resultados e benefícios com esses investimentos surgem. Em geral, trabalhos de revisão da teoria de projetos apresentam, entre outras, as seguintes perguntas (IKA, 2009; SÖDERLUND, 2004):

- Como provar que gastar dinheiro em métodos e capacitação em gerenciamento de projetos tem valor e qual a função ou valor adicionado pela gestão de projetos?

- Como tornar os altos executivos comprometidos com os métodos de gerenciamento de projetos sem mostrar os resultados financeiros gerados em decorrência dos investimentos realizados?

- O que determina o sucesso ou a falha de um projeto?

As poucas evidências quantitativas existentes apresentam um cenário nada animador. $O$ The Standish Group (2009), baseando-se em uma pesquisa com 280.000 projetos de tecnologia de informação conduzida em 2008, mostra que apenas 32\% dos projetos podem ser considerados um sucesso. Próximo da metade dos projetos pesquisados, isto é $44 \%$, apresenta problemas de prazos 
ou custos e $24 \%$ já não podem mais ser recuperados e foram considerados projetos com falhas. Segundo a mesma pesquisa, os projetos que gastam mais do que o valor previsto, estouram seu orçamento, em média, em $45 \%$. Quanto a completar o projeto no prazo, os dados da pesquisa também não são nada animadores, em média, o cronograma é ampliado em $63 \%$ de seu prazo original, sendo que somente $67 \%$ das características requeridas e funcionalidades são normalmente entregues aos clientes dos projetos. Os projetos falham e isto não vai mudar, a menos que as empresas comecem a medir aonde os projetos falham e por que (BUCHANAN, 2008).

Apesar do volume de literatura existente na área, ainda há poucas evidências empíricas que relacionem implementação de gerenciamento de projetos aos resultados obtidos. Por outro lado, os executivos também buscam evidências de que seus investimentos estão funcionando efetivamente e produzindo o valor esperado de lucros ao final dos projetos. Em pesquisas recentes sobre a medição do valor e do sucesso do gerenciamento de projetos, a questão de se quantificar seu valor ainda não se encontra satisfatoriamente respondida (IKA, 2009; THOMAS; MULLALY, 2008).

Com o objetivo de contribuir para a avaliação dos benefícios do gerenciamento de projetos nas organizações, este trabalho busca avaliar a relação entre o grau de implementação de uma metodologia de gerenciamento de projetos, incluindo o desenvolvimento e uso de ferramentas e métodos, e os resultados dos projetos. Como proxy de retorno obtido será utilizado o desempenho operacional alcançado pelos projetos. A abordagem metodológica envolve um estudo longitudinal, com estratégia híbrida, qualitativa e quantitativa.

Este artigo está estruturado em 5 seções. A seção 2 traz a síntese do quadro teórico, seguida da seção 3 que apresenta a abordagem metodológica. Os resultados e as discussões são apresentados na seção 4. Por fim, a seção 5 traz as conclusões e recomendações para trabalhos futuros.

\section{REVISÃO DE LITERATURA}

\subsection{MÉTODOS DE GERENCIAMENTO DE PROJETOS}

Projetos podem ser definidos como organizações "esquecíveis", que surgem em meio à rotina, sendo compostas por indivíduos que dificilmente irão trabalhar juntos novamente. Uma estratégia para codificar as capacidades da organização em gestão de projetos, deve ser definida no nível da empresa para repetir abordagens de sucesso em próximos projetos. Utilizando-se um

Revista de Gestão e Projetos - GeP, São Paulo, v. 3, n. 2, p 178-206, mai./ago. 2012. 
processo bem estruturado e implementado, estas capacidades podem ser armazenadas e transferidas ao longo do tempo, do espaço e do contexto. Adicionalmente, por meio da criação de uma memória externa aos indivíduos, este tipo de codificação de conhecimento pode tornar as organizações menos vulneráveis à perda do conhecimento tácito armazenado nas pessoas (IBERT, 2004).

Uma sistemática de projetos pode ser composta por métodos, pacotes de ferramentas e modelos de projetos. Desse modo, a gestão de projetos pode ser vista como a aplicação sequencial de processos estruturados, repetidos e contínuos que, quando utilizados por uma organização de forma gradual e segura para seus negócios, permite dar passos rumo à institucionalização de práticas padronizadas.

Além disso, a sistemática precisa auxiliar a equipe no planejamento e entrega dos projetos, considerando o ciclo inteiro de vida, de forma consistente e eficiente, sempre orientada para os negócios e para a satisfação dos clientes (SILVEIRA, 2008). E para isso, há algumas características das sistemáticas de gerenciamento de projetos das organizações destacadas pelo autor, como estar em sintonia com a ISO 9000 ou com outras normas de institutos oficiais de gerenciamento de projetos. Deve também ter um conjunto compreensivo e flexível de processos, ferramentas e técnicas que dêem suporte às atividades, auditadas periodicamente por um Escritório de Gestão de Projetos (Project Management Office - PMO). Ressalta ainda a necessidade de documentação, instrumentos de medição e controle de projetos durante o ciclo de vida e comunicação dos resultados aos stakeholders.

Existem, atualmente, vários conjuntos de modelos de métodos de gerenciamento de projetos, disponíveis para utilização por profissionais e organizações para melhor gerenciar seus projetos. Os métodos atualmente mais difundidos são disponibilizados por institutos e associações dedicados ao estudo de projetos conforme apresentado no Quadro 1.

Revista de Gestão e Projetos - GeP, São Paulo, v. 3, n. 2, p 178-206, mai./ago. 2012. 


\begin{tabular}{|c|c|c|c|}
\hline Instituto & Conjunto de Métodos & $\begin{array}{l}\text { País de } \\
\text { Origem }\end{array}$ & Foco da Metodologia \\
\hline $\begin{array}{l}\text { Project Management } \\
\text { Institute }(\mathrm{PMI})\end{array}$ & $\begin{array}{l}\text { Project Management } \\
\text { Body of Knowledge } \\
\text { (PMBoK) }\end{array}$ & EUA & Gestão geral de projetos \\
\hline $\begin{array}{l}\text { International Project } \\
\text { Management Association } \\
\text { (IPMA) }\end{array}$ & $\begin{array}{l}\text { ICB - IPMA } \\
\text { Competence Baseline }\end{array}$ & $\begin{array}{c}\text { União } \\
\text { Européia }\end{array}$ & Gestão geral de projetos \\
\hline $\begin{array}{l}\text { Australian Institute of } \\
\text { Project Management } \\
\text { (AIPM) }\end{array}$ & $\begin{array}{l}\text { AIPM - Professional } \\
\text { Competency Standards } \\
\text { for Project Management }\end{array}$ & Austrália & Gestão geral de projetos \\
\hline $\begin{array}{l}\text { Association for Project } \\
\text { Management (APM) }\end{array}$ & $\begin{array}{l}\text { APM Body of } \\
\text { Knowledge }\end{array}$ & Reino Unido & Gestão geral de projetos \\
\hline $\begin{array}{l}\text { Office of Government } \\
\text { Commerce (OGC) }\end{array}$ & $\begin{array}{l}\text { Projects In Controlled } \\
\text { Environments } \\
\text { (PRINCE2) }\end{array}$ & Reino Unido & $\begin{array}{c}\text { Gestão de projetos de } \\
\text { sistemas de informação }\end{array}$ \\
\hline $\begin{array}{l}\text { Japan Project Management } \\
\text { Forum (JPMF) }\end{array}$ & $\begin{array}{l}\text { ENAA Model Form- } \\
\text { International Contract } \\
\text { for Process Plant } \\
\text { Construction }\end{array}$ & Japão & $\begin{array}{l}\text { Gestão de projetos de } \\
\text { construções }\end{array}$ \\
\hline
\end{tabular}

Quadro 1 - Principais associações de gerenciamento de projetos e seus conjuntos de métodos.

De modo geral, o que se observa é que o PMBoK é um conjunto de métodos genérico e bastante abrangente que objetiva atender às necessidades dos mais diversos tipos de projetos (PMI, 2008). Por isso mesmo, deve ser adaptado quando da aplicação de seus conceitos por parte das organizações. Durante a adaptação, é grande a chance da organização se perder no grau de profundidade com que deve tratar os 42 processos presentes no documento, frente às suas necessidades específicas. Além disso, se a organização também conduz programas, deve buscar os conceitos adicionais e específicos na norma de programa publicada pelo PMI.

A norma européia de gerenciamento de projetos, publicada pelo IPMA, o ICB, se configura como uma excelente fonte de referência para aqueles que buscam uma opção de métodos em gerenciamento de projetos mais voltada para os aspectos humanos da gestão (IPMA, 2006). Estruturado por competências que o projeto necessita desenvolver, seu conteúdo encontra-se dividido em três partes: competências contextuais, comportamentais e técnicas.

Outros dois guias de referência de associações ainda pouco difundidas em organizações brasileiras são o australiano AIPM (AIPM, 2008) e o inglês APM Body of Knowledge (APM, 2006). O guia do instituto australiano de projetos configura-se como uma boa fonte de referência para os aspectos humanos do gerenciamento de projetos. O instituto inglês de gerenciamento de projetos apresenta um dos mais completos conjuntos de métodos pesquisado neste trabalho. Além dos 
aspectos relacionados à gestão técnica de projetos, este documento apresenta conteúdos que tratam os conceitos do valor do gerenciamento de projetos, dos modelos e sistemáticas de implementação de escritórios de projetos e dos aspectos estratégicos da gestão de projetos.

Mais voltado ao mercado de tecnologia de informação, o PRINCE 2, apresenta-se estruturado por etapas de um projeto e por atividades a serem conduzidas pela equipe de gestão do mesmo, por isso é um pouco mais voltado à aplicação prática do que os demais (OGC, 1996).

O ENAA, publicado pelo instituto japonês de gerenciamento de projetos, dedica-se a analisar com mais profundidade os aspectos técnicos e contratuais de grandes projetos de engenharia tendo, portanto, uma aplicação mais restrita a determinados segmentos de mercado (ENAA, 1992).

O Quadro 2 apresenta de uma forma resumida, os conjuntos de métodos em gerenciamento de projetos, suas principais características e diferenças entre os demais.

\begin{tabular}{|c|c|c|}
\hline Conjunto de Métodos & Características & Diferenças entre os Demais \\
\hline $\begin{array}{l}\text { PMBoK - Project } \\
\text { Management Body of } \\
\text { Knowledge }\end{array}$ & $\begin{array}{l}\text { Conjunto de métodos desenvolvido para } \\
\text { diversos tipos de projetos, sendo, } \\
\text { portanto, bastante genérico. Estruturado } \\
\text { por áreas de conhecimento de um } \\
\text { projeto. }\end{array}$ & $\begin{array}{c}\text { É complementado por dois } \\
\text { conjuntos de métodos adicionais: } \\
\text { Programa e Portfólio. }\end{array}$ \\
\hline $\begin{array}{l}\text { ICB - IPMA Competence } \\
\text { Baseline }\end{array}$ & $\begin{array}{l}\text { Estruturado por competências que o } \\
\text { projeto necessita desenvolver, divididas } \\
\text { em: contextuais, comportamentais e } \\
\text { técnicas. }\end{array}$ & $\begin{array}{c}\text { Juntamente com a norma } \\
\text { australiana, apresenta um grau de } \\
\text { profundidade bem maior que os } \\
\text { demais métodos nos aspectos } \\
\text { humanos da figura do gerente do } \\
\text { projeto. }\end{array}$ \\
\hline $\begin{array}{l}\text { AIPM Professional } \\
\text { Competency Standards for } \\
\text { Project Management }\end{array}$ & $\begin{array}{c}\text { Este documento, publicado pelo } \\
\text { instituto australiano de projetos, é } \\
\text { bastante similar em sua estrutura ao } \\
\text { PMBoK, dividido por áreas de } \\
\text { conhecimento. }\end{array}$ & $\begin{array}{l}\text { Também possui um enfoque mais } \\
\text { profundo nas habilidades humanas. }\end{array}$ \\
\hline APM Body of Knowledge & $\begin{array}{l}\text { Um dos mais completos conjuntos de } \\
\text { métodos, este documento apresenta } \\
\text { conteúdos relacionados a projetos, } \\
\text { valor, escritório de projetos e aspectos } \\
\text { estratégicos da gestão de projetos. }\end{array}$ & $\begin{array}{c}\text { É o mais abrangente dos conjuntos } \\
\text { de métodos. }\end{array}$ \\
\hline $\begin{array}{l}\text { PRINCE2 - Projects In } \\
\text { Controlled Environments }\end{array}$ & $\begin{array}{l}\text { Conjunto de métodos estruturado por } \\
\text { etapas de um projeto e nas atividades a } \\
\text { serem conduzidas pela equipe de gestão } \\
\text { do mesmo. }\end{array}$ & $\begin{array}{c}\text { Conjunto de métodos mais voltado } \\
\text { para projetos de tecnologia de } \\
\text { informação. }\end{array}$ \\
\hline $\begin{array}{l}\text { ENAA Model Form- } \\
\text { International Contract for } \\
\text { Process Plant } \\
\text { Construction }\end{array}$ & $\begin{array}{l}\text { O documento tem um enfoque muito } \\
\text { grande nos aspectos contratuais de um } \\
\text { projeto. }\end{array}$ & $\begin{array}{c}\text { O foco deste conjunto de métodos } \\
\text { são projetos de construção em } \\
\text { engenharia. }\end{array}$ \\
\hline
\end{tabular}

Quadro 2 - Os conjuntos de métodos em gerenciamento de projetos e suas principais características.

Revista de Gestão e Projetos - GeP, São Paulo, v. 3, n. 2, p 178-206, mai./ago. 2012. 


\subsection{DESEMPENHO EM PROJETOS}

A efetividade do processo de gerenciamento de projetos irá determinar se os projetos desempenham um forte papel em fornecer uma fonte de vantagem competitiva para uma organização (BARBER, 2004).

Mesmo as organizações que aparentemente apresentam sucesso nos seus projetos, possuem uma inconsistência latente do mesmo e uma falta de habilidade para identificar o problema e a maneira de reduzi-lo (BUCHANAN, 2008). Um primeiro passo para resolver o problema é padronizar o que significa uma performance satisfatória e que o inimigo natural de uma performance de excelência consistente é a disparidade. Para Buchanan (2008), em geral as métricas se dividem em duas categorias: medidas preditivas que ajudam a prever ou identificar tendências funcionando como um sistema de alerta inicial e medidas corretivas que auxiliam a estabelecer padrões para melhorar a performance em projetos futuros.

Diversos trabalhos têm sido conduzidos, nesta última década, buscando analisar como o sucesso em projetos pode ser medido (BELOUT; GAUVREAU, 2004; BESNER; HOBBS, 2006; BIZAN, 2003; DVIR; RAZ; SHENHAR, 2003; GRAY, 2001; KENDRA; TAPLIN, 2004; LIPOVETSKY et al., 2005; RAZ; SHENHAR; DVIR, 2002; REPISO; SETCHI; SALMERON, 2007).

O sucesso em projetos é usualmente definido como cumprir os objetivos de tempo, custo e qualidade e satisfazer os stakeholders do projeto.

Apesar disso, as pesquisas conduzidas, ao longo dos últimos anos, e desde os anos 1980, que vêm investigando as dimensões do sucesso em projetos, levaram a re-escrever a fórmula acima com a concordância geral de que o sucesso em projetos é multi-dimensional e que diferentes pessoas medem o sucesso de projetos em diferentes maneiras em épocas diferentes (BARBER, 2004; BRYDE, 2003; IKA, 2009; JUGDEV; MULLER, 2005).

Com o objetivo de se medir o sucesso em projetos é possível criar critérios e métricas como proposto por Ling (2004), que faz uma divisão refinando o sucesso em projetos, em atingir sucesso no produto do projeto, através do atendimento de padrões de qualidade e atingir sucesso no processo por meio do cumprimento de objetivos de tempo e orçamento. Para isso, são utilizadas quatro métricas para avaliar a performance dos projetos: custo, tempo, qualidade e atendimento da satisfação do cliente.

Já Larson e Gobeli (1989) apresentam alguns fatores que podem afetar o sucesso de

Revista de Gestão e Projetos - GeP, São Paulo, v. 3, n. 2, p 178-206, mai./ago. 2012. 
projetos, tais como: estrutura do projeto, competência do gerente do projeto e tamanho do projeto, utilizando os mesmos indicadores propostos por Ling (2004).

Métricas trazem consistência e formalidade à gestão de projetos. Com métricas, decisões importantes de projeto podem ser tomadas com base em informações. Em essência, métricas trazem objetividade às ferramentas para o monitoramento do progresso de projetos e ajudam melhorar a organização, por permitir uniformidade, acuracidade e repetibilidade (RAD; LEVIN, 2006). Com este intuito Rad e Levin (2006) propõe métricas para a empresa, para as pessoas e para o próprio projeto.

Outra maneira de se avaliar o sucesso é desdobrá-lo em dois critérios diferentes. Um, o próprio sucesso, que segundo Cooke-Davies (2002), não pode ser medido até que o projeto seja finalizado, e outro, a performance de projetos, que pode ser medida durante a execução. Segundo o autor, nenhum sistema de métricas em projetos pode ser considerado completo, sem um dos pacotes de medidas (performance e sucesso) e deve-se buscar um método de ligá-los, como um meio de avaliar a acuracidade, com a qual a performance dos projetos prediz o sucesso da organização.

Thamhain (2004) em um estudo conduzido, entre 2000 e 2003, com 76 times de projetos de 27 empresas, busca, por sua vez, associar o ambiente de equipes de projetos com a performance dos mesmos. Segundo o autor, as principais variáveis relacionadas aos times de projetos que influenciam o sucesso, são o ambiente do time e a performance da equipe.

Os benefícios gerados para o cliente (DVIR et al., 1998), a adaptabilidade e a habilidade do projeto em cooperar com outras áreas da organização (KATZ; ALLEN, 1985) e o atendimento de padrões de qualidade e segurança (WHITE; FORTUNE, 2002), também, devem ser inclusos em sistemas de medição de desempenho de projetos.

Critérios financeiros vêem sendo utilizados para se medir a performance em projetos já há algum tempo. Os critérios podem incluir retorno econômico, análise custo/benefício (ARCHER; GHASEMZADEH, 1999), contribuição da melhoria de medidas financeiras tais como lucros, market share e valor de novos projetos obtidos (THOMAS; DELISLE; JUGDEV, 2002).

Outra maneira direta de se avaliar os benefícios do gerenciamento de projetos é analisar as margens dos projetos correntes de uma empresa. É possível comparar a margem de um projeto quando a empresa o vende, com o valor obtido quando ele é concluído. A diferença pode ser explicada em parte pela metodologia de gerenciamento de projetos (PATAH; CARVALHO, 2007).

Para ter um valor real, medidas de performance devem ser cuidadosamente alinhadas com objetivos organizacionais claros, tais como eficiência, produtividade ou previsibilidade. Porém, a 
simples presença de um sistema de medição, que pode nunca ser perfeito, irá causar melhorias na performance dos projetos. Quando alguém decide que alguma atividade necessita ser medida, então ela se torna importante e as pessoas começam a se preocupar com a mesma. E este fato, mesmo que isoladamente, irá por si só, melhorar a performance dos projetos (BUCHANAN, 2008).

Para a medição da performance dos projetos neste trabalho, os critérios de medição de desempenho são divididos em operacionais e estratégicos. Os critérios de desempenho operacional são aqueles relacionados a atingir sucesso no produto do projeto, através do atendimento de padrões de qualidade, e atingir sucesso no processo de gestão do projeto, através do cumprimento de objetivos de tempo e orçamento. Os critérios de desempenho estratégico são decorrentes de definições externas aos projetos, relacionadas com o ambiente organizacional em que o projeto está inserido, em que se busca atingir o sucesso esperado pelo cliente e outros stakeholders do projeto.

O Quadro 3 apresenta o resumo das métricas para medição de performance em projetos, pesquisadas em várias fontes para a elaboração deste trabalho. 


\begin{tabular}{|c|c|}
\hline Autor & Métricas para Medição de Performance em Projetos \\
\hline Katz e Allen (1985) & $\begin{array}{l}\text { - Cronograma; } \\
\text { - Orçamento; } \\
\text { - Performance de custo; } \\
\text { - Inovação no projeto; } \\
\text { - Adaptabilidade; } \\
\text { - Habilidade em cooperar com outras áreas da organização. }\end{array}$ \\
\hline Larson e Gobeli (1989) & $\begin{array}{l}\text { - Controle dos custos; } \\
\text { - Cumprimento de prazos; } \\
\text { - Performance técnica. }\end{array}$ \\
\hline Markowitz (1990) & - Incerteza. \\
\hline Dvir et al. (1998) & $\begin{array}{l}\text { - Cumprimento das metas definidas; } \\
\text { - Benefícios para o cliente. }\end{array}$ \\
\hline Archer e Ghasemzadeh (1999) & $\begin{array}{l}\text { - Retorno econômico; } \\
\text { - Análise custo/benefício; } \\
\text { - Riscos; } \\
\text { - Impacto no mercado. }\end{array}$ \\
\hline Gray (2001) & $\begin{array}{l}\text { - Orçamento; } \\
\text { - Cronograma; } \\
\text { - Especificação técnica; } \\
\text { - Opinião dos stakeholders. }\end{array}$ \\
\hline White e Fortune (2002) & $\begin{array}{l}\text { - Requerimentos de clientes atendidos; } \\
\text { - Completado dentro do cronograma; } \\
\text { - Completado dentro do orçamento; } \\
\text { - Objetivos organizacionais atendidos; } \\
\text { - Negócios fortalecidos; } \\
\text { - Descontinuidades nos negócios minimizadas; } \\
\text { - Padrões de qualidade e segurança atendidos. }\end{array}$ \\
\hline Ibbs e Reginato (2002) & - Valor medido pelo processo. \\
\hline Thomas, Delisle e Jugdev (2002) & $\begin{array}{l}\text { - Contribuição da melhoria de medidas financeiras; } \\
\text { - Melhoria do desempenho das equipes de projetos; } \\
\text { - Melhoria da satisfação dos consumidores, dos lucros e do } \\
\text { market share; } \\
\text { - Valor de novos projetos obtidos. }\end{array}$ \\
\hline Ling (2004) & $\begin{array}{l}\text { - Custo; } \\
\text { - Tempo; } \\
\text { - Qualidade; } \\
\text { - Atendimento da satisfação do cliente. }\end{array}$ \\
\hline Thamhain (2004) & $\begin{array}{l}\text { - Ambiente do time do projeto; } \\
\text { - Performance do time do projeto. }\end{array}$ \\
\hline Rad e Levin (2006) & $\begin{array}{l}\text { - Empresa; } \\
\text { - Pessoas; } \\
\text { - Coisas. }\end{array}$ \\
\hline Patah e Carvalho (2007) & $\begin{array}{l}\text { - Medida da comparação entre o custo de um projeto quando a } \\
\text { companhia inicia o mesmo com o valor obtido quando o projeto é } \\
\text { concluído. }\end{array}$ \\
\hline
\end{tabular}

Quadro 3 - Métricas para medição de performance em projetos.

Revista de Gestão e Projetos - GeP, São Paulo, v. 3, n. 2, p 178-206, mai./ago. 2012. 
Pode-se observar que às métricas para medição da performance em projetos mais utilizadas são aquelas relacionadas à obtenção, ao final do projeto, dos valores inicialmente planejados de prazo e custo (GRAY, 2001; KATZ; ALLEN, 1985; LARSON; GOBELI, 1989; LING, 2004; WHITE; FORTUNE, 2002), sendo que é consenso a questão financeira envolvida (ARCHER; GHASEMZADEH, 1999; PATAH; CARVALHO, 2007; THOMAS; DELISLE; JUGDEV, 2002), o que já era esperado. Alguns destes autores, porém, incluem outros conceitos como riscos (ARCHER; GHASEMZADEH, 1999), satisfação de stakeholders, novos projetos obtidos e desempenho da equipe (THOMAS; DELISLE; JUGDEV, 2002). E outros, fornecem uma visão um pouco distinta ao incluir o valor, como uma função dos processos que as organizações geram (IBBS; REGINATO, 2002).

\section{METODOLOGIA E HIPÓTESES}

\subsection{MODELO CONCEITUAL}

Vários fatores podem levar a um alto desempenho em projetos, como visto anteriormente. Neste estudo, enfatiza-se o uso de métodos de gerenciamento de projetos (KESSLER; WINKELLHOFER, 2002; THE STANDISH GROUP, 2009; WHITE; FORTUNE, 2002). Desta forma, foi selecionado o seguinte aspecto para análise neste trabalho:

- Possuir um sistema de gerenciamento de projetos formal com métodos bem definidos.

Dos indicadores de medição do desempenho discutidos na seção anterior, são considerados neste trabalho:

- Cumprimento do orçamento planejado;

- Cumprimento do prazo planejado;

- Performance financeira do projeto relacionada às análises e decisões de investimentos realizadas antes da aprovação do projeto.

Revista de Gestão e Projetos - GeP, São Paulo, v. 3, n. 2, p 178-206, mai./ago. 2012. 


\subsection{HIPÓTESE DA PESQUISA - USO DE MÉTODOS DE GERENCIAMENTO DE PROJETOS}

Vários autores enumeram os benefícios do uso de métodos de gerenciamento de projetos (IBERT, 2004; KERZNER, 2001; KESSLER; WINKELLHOFER, 2002; THE STANDISH GROUP, 2009; WHITE; FORTUNE, 2002). Kerzner (2001) apresenta uma lista de benefícios, entre eles: a melhoria do cumprimento das atividades em relação aos planos e o atendimento aos objetivos do projeto. Ibert (2004), quando comenta as características dos projetos, apresenta o conceito que o uso de métodos padronizados de gestão de projetos, pode fazer com que a empresa repita abordagens de sucesso em próximos projetos. Com o objetivo de medir quantitativamente o impacto da adoção de métodos de gerenciamento de projetos na performance dos mesmos, a primeira hipótese deste trabalho é formulada da seguinte maneira:

- H01: O grau de utilização de métodos de gerenciamento de projetos não influencia o resultado operacional dos projetos.

Derivam desta segunda hipótese as seguintes hipóteses:

- H01a: O grau de utilização de métodos de gerenciamento de projetos não influencia no cumprimento do prazo dos projetos.

- H01b: O grau de utilização de métodos de gerenciamento de projetos não influencia no cumprimento do custo.

- H01c: O grau de utilização de métodos de gerenciamento de projetos não influencia na performance financeira dos projetos.

Todas as hipóteses nulas contêm sempre uma igualdade sobre a qual se deve obter evidências para rejeitá-la. O nível de significância $(\alpha)$, fixado neste estudo em 5\%, representa a probabilidade (ou risco) de se incorrer no erro de rejeitar H0, quando esta hipótese é de fato verdadeira. 


\subsection{SELEÇÃO DA AMOSTRA DE ANÁLISE}

Conforme sugerido por Eisenhardt e Graebner (2007), foi utilizada a amostragem teórica (theoretical sampling). Como o foco da pesquisa são organizações em que a atividade de projetos é estratégica, e, por conseguinte, investem grandes montas em métodos e processos em gestão de projetos, esse foi o recorte teórico utilizado para definição da amostra. Em trabalhos que estudaram a performance em projetos, pouca evidência foi encontrada em organizações com pouco histórico de gestão de projetos (BRYDE, 2003; SILVEIRA, 2008).

Além disso, para a análise longitudinal, tinha-se como requisito o acesso aos dados de desempenho do projeto em uma base temporal significativa para permitir a comparação do desempenho dos projetos antes, durante e após a implantação dos métodos, em uma abordagem similar à utilizada por Hendricks e Singhal (2001). Dados para tratar as questões de valores em projetos não se encontram facilmente disponíveis na maioria das organizações (THOMAS; MULLALY, 2008). Ao se optar por uma única organização, com amplo acesso às informações, busca-se reduzir sensivelmente este risco de disponibilidade de informações normalmente tratadas como confidenciais.

Por fim, necessitava-se de uma base comum para comparação do avanço da implantação, do uso dos métodos e da maturidade em projetos. Dada a grande quantidade de diferentes métodos disponíveis para a gestão de projetos, conforme apresentado na seção 2, caso a escolha recaísse sobre várias organizações, seria necessário equalizar estes diversos métodos adotados ou adaptados pelas empresas, para obter uma base comparável de variáveis independentes. Além disso, em decorrência das inúmeras possibilidades de medição do sucesso em projetos, também discutidas na seção 2, seria necessário gerar uma base comparável para as variáveis dependentes, conforme apresentado por Pinto (2002).

A organização estudada é uma empresa multinacional com diversas divisões atuando em diferentes mercados, em que foi possível obter dados de uma grande quantidade de projetos por um longo período de análise. Essa empresa tem $60 \%$ de suas vendas brutas provenientes de projetos. A empresa produz e instala uma grande variedade de equipamentos, na sua maioria, entregues aos clientes por meio de projetos específicos, adaptados às necessidades de cada um. A taxa de inovação dos produtos é extremamente alta, e os produtos atualmente vendidos foram desenvolvidos no máximo três anos atrás. Basicamente, a empresa vende soluções customizadas para seus clientes, com baixo volume de unidades produzidas e uma grande variedade.

Revista de Gestão e Projetos - GeP, São Paulo, v. 3, n. 2, p 178-206, mai./ago. 2012. 
O programa de implementação de gestão de projetos nas unidades de negócio da empresa na região do Mercosul data de 2003, com o início dos cursos de certificação, estruturação do PMO e difusão da metodologia da matriz. Desde então, a sistemática de compartilhamento das melhores práticas, a criação de padrões em gerenciamento de projetos, a busca de um número suficiente de gerentes de projeto qualificados e a uniformização da cultura de gerenciamento de projetos na empresa foram feitas visando atingir a rentabilidade sustentada nas operações.

A empresa tem diversas unidades de negócio, e para o dimensionamento da amostra de projetos analisados foi adotada a regra prática proposta por Hair et al. (1998), de que quando se trata da análise de dependência ou interdependência entre variáveis, deve-se obter, pelo menos, 20 amostras para cada variável estudada, o que totalizou 120 amostras de projetos a serem obtidas durante a etapa de coleta de dados.

\subsection{OPERACIONALIZAÇÃO DAS VARIÁVEIS DA PESQUISA}

O sucesso dos projetos foi medido com o objetivo de avaliar o benefício gerado, por meio do uso de métodos em gerenciamento de projetos por parte da equipe que gerencia o mesmo e, também, mais especificamente do gerente do projeto. Procura-se, com isso, medir a influência que o esforço em gerenciamento de projetos gera na obtenção de um maior retorno financeiro pelas empresas. Espera-se que esta correlação permita medir a melhoria no desempenho de uma empresa pelo uso do gerenciamento de projetos. Para todos os projetos foram obtidas as informações contidas no Anexo 1.

O esforço na implementação de gerenciamento de projetos envolve o desenvolvimento e uso de ferramentas e métodos, e suporte administrativo e organizacional. Como proxy de retorno foi utilizado o desempenho operacional obtido pelos projetos, avaliado através dos critérios de resposta.

O grau de implementação dos métodos de gestão de projetos é avaliado nas diversas unidades de negócio da empresa em uma escala de 0 a 100\%, por um departamento de auditoria central.

O presente trabalho define variáveis independentes e dependentes segundo o conceito de Marconi e Lakatos (2003).

Como a variável independente (X) é aquela que influencia, determina ou afeta outra variável, de acordo com as hipóteses, foi escolhida a variável independente de uso de métodos de gerenciamento de projetos.

Revista de Gestão e Projetos - GeP, São Paulo, v. 3, n. 2, p 178-206, mai./ago. 2012. 
Uma vez que a variável dependente (Y) consiste naqueles valores (fenômenos ou fatores) a serem explicados ou descobertos, em virtude de serem influenciados, determinados ou afetados pela variável independente, a escolha do cumprimento de custos, prazos e a performance financeira do projeto, baseou-se nos principais indicadores de medição de desempenho selecionados da teoria, conforme discutido anteriormente.

Neste trabalho, a variável independente é medida contra as variáveis dependentes, de acordo com a hipótese 01 .

O Quadro 4 apresenta as variáveis selecionadas para este trabalho e suas respectivas formas de medição e grandeza.

\begin{tabular}{|c|c|c|}
\hline VARIÁVEL & FORMA DE MEDIÇÃo & GRANDEZA \\
\hline $\begin{array}{c}\text { X1 - Uso de Métodos de } \\
\text { Gerenciamento de Projetos }\end{array}$ & $\begin{array}{c}\text { Grau de implementação de gerenciamento de } \\
\text { projetos onde se busca medir a proporção em } \\
\text { que os métodos estão introduzidos e são } \\
\text { utilizados. }\end{array}$ & $\%$ \\
\hline Y1 - Cumprimento de Orçamento & $\begin{array}{c}\text { Variação relativa do aumento de orçamento, } \\
\text { medida pela diferença em valor monetário do } \\
\text { orçamento original previsto pelo projeto em } \\
\text { relação ao orçamento real final do projeto, } \\
\text { dividida pelo orçamento original. }\end{array}$ & $\%$ \\
\hline Y2 - Cumprimento de Prazo & $\begin{array}{c}\text { Variação relativa do aumento de prazo do } \\
\text { projeto, medida através da diferença entre o } \\
\text { prazo original planejado em dias e o prazo } \\
\text { final do projeto, dividida pelo prazo original. }\end{array}$ \\
\hline Y3 - Performance Financeira & $\begin{array}{c}\text { Variação relativa da margem do projeto, } \\
\text { medida através da diferença entre a margem } \\
\text { final do projeto e a margem prevista } \\
\text { originalmente, dividida pela margem } \\
\text { original. }\end{array}$ & $\%$ \\
\hline
\end{tabular}

Quadro 4 - Variáveis e formas de medição.

O Quadro 5 apresenta a hipótese e os resultados esperados das relações entre a variável independente e as variáveis dependentes.

Revista de Gestão e Projetos - GeP, São Paulo, v. 3, n. 2, p 178-206, mai./ago. 2012. 


\begin{tabular}{|c|c|c|c|}
\hline HIPÓTESE & $\begin{array}{c}\text { VARIÁVEL } \\
\text { INDEPENDENTE (X) }\end{array}$ & VARIÁVEL DEPENDENTE (Y) & $\begin{array}{c}\text { RESULTADO } \\
\text { ESPERADO }\end{array}$ \\
\hline \multirow{3}{*}{ H01 } & X1 - Uso de Métodos de & Y1 - Cumprimento de Orçamento \\
& Gerenciamento de Projetos & Y3 - Cumprimento de Prazo & Influencia \\
& & & \\
\hline
\end{tabular}

Quadro 5 - Hipótese de pesquisa e influências a serem verificadas.

Para a análise dos dados obtidos utilizou-se um software específico de análise estatística que atendia plenamente as necessidades do trabalho, o Statistical Package for the Social Sciences (SPSS).

\section{DISCUSSÕES E ANÁLISE DOS RESULTADOS}

Foram obtidos dados de 2851 projetos conduzidos no período de janeiro de 2005 a junho de 2008. Porém, vários destes projetos não apresentavam informações completas como os custos finais de execução, a categorização atribuída e outros. Após uma análise detalhada, chegou-se a 1387 projetos com dados completos, ou 48,65\% do total, possíveis de serem analisados, para se tentar comprovar a hipótese estabelecida na seção 3. Estes projetos foram conduzidos entre julho de 2006 e junho de 2008. Para todos os projetos foram obtidas as informações contidas no Anexo 1.

Em relação ao segmento de atuação do projeto, a área com mais projetos obtidos é a área de energia, com 713 projetos $(51,41 \%$ do total), seguida pela área de medicina com 218 projetos. Quanto ao país de realização, a maior parte dos projetos foi conduzida no Brasil, com 823 projetos (59,34\% do total), seguido da Argentina, com 294 projetos $(21,20 \%)$ e do Chile, com 270 projetos $(19,47 \%)$.

\subsection{VARIÁVEL INDEPENDENTE}

A variável independente Uso de Métodos de Gerenciamento de Projetos (X1) está concentrada entre $70,83 \%$ e $83,33 \%$ e apresenta uma baixa dispersão relativa (coeficiente de variação $=4 \%$ ), conforme Tabela 1 .

Revista de Gestão e Projetos - GeP, São Paulo, v. 3, n. 2, p 178-206, mai./ago. 2012. 
Tabela 1 - Distribuição de frequência da variável independente X1.

\begin{tabular}{|c|c|c|}
\hline GRAU DE IMPLANTAÇÃO & FREQUÊNCIA & $\%$ \\
\hline $70,83 \%$ & 71 & 5,12 \\
\hline $73,81 \%$ & 122 & 8,80 \\
\hline $75,00 \%$ & 294 & 21,20 \\
\hline $75,93 \%$ & 162 & 11,68 \\
\hline $75,98 \%$ & 178 & 12,83 \\
\hline $77,60 \%$ & 150 & 10,81 \\
\hline $80,83 \%$ & 137 & 9,88 \\
\hline $80,95 \%$ & 110 & 7,93 \\
\hline $82,64 \%$ & 87 & 6,27 \\
\hline $83,33 \%$ & 76 & 5,48 \\
\hline Total & 1387 & 100,00 \\
\hline
\end{tabular}

De acordo com esta variável, os projetos têm média de 77,18\% de grau de implantação, mediana de $75,98 \%$ e moda de $75,00 \%$, e desvio padrão de $3,29 \%$.

\subsection{VARIÁVEIS DEPENDENTES}

A variável dependente Y1 - Cumprimento de Orçamento distribuí-se num intervalo entre mínimo de $-100 \%$ (valor final maior do que valor estimado) e 716,6\% (valor final menor do que valor estimado). Adicionalmente, quase metade dos projetos empata o custo final com o planejado e

Revista de Gestão e Projetos - GeP, São Paulo, v. 3, n. 2, p 178-206, mai./ago. 2012. 
poucos projetos $(12,98 \%)$ têm seu custo final acima do planejado, conforme Tabela 2.

Tabela 2 - Distribuição de frequência da variável dependente Y1.

\begin{tabular}{|c|c|c|}
\hline ÍNDICE DE VARIAÇÃO RELATIVA DE CUSTO & FREQUÊNCIA & \% \\
\hline Negativo & 180 & 12,98 \\
\hline Zero & 667 & 48,09 \\
\hline Positivo & 540 & 38,93 \\
\hline Total & 1387 & 100,00 \\
\hline
\end{tabular}

Os projetos têm uma variação média de custo de 9,51\% abaixo do estimado, com mediana e moda igual a zero. A dispersão relativa (dispersão cerca de quase 5 vezes a média) é alta, com coeficiente de variação de $495 \%$ e desvio-padrão de $47,07 \%$.

A variável dependente Y2 - Cumprimento de Prazo distribuí-se num intervalo entre -10\% (projeto atrasado) e $+10 \%$ (projeto adiantado). A maioria dos projetos $(70,23 \%)$ é finalizada dentro da data planejada. Os valores são apresentados na Tabela 3.

Tabela 3 - Distribuição de frequência da variável dependente Y2.

\begin{tabular}{|c|c|c|}
\hline ÍNDICE DE VARIAÇÃO RELATIVA DE PRAZO & FREQUÊNCIA & $\%$ \\
\hline Negativo & 412 & 29,70 \\
\hline Zero & 1 & 0,07 \\
\hline Positivo & 974 & 70,23 \\
\hline Total & 1387 & 100,00 \\
\hline
\end{tabular}

Os projetos têm prazo final, em média de 4,66\% abaixo do esperado, mediana e moda de 9,8\% abaixo do inicialmente estimado. A dispersão relativa é alta com coeficiente de variação de 
$159 \%$ e desvio-padrão de $7,42 \%$.

A variável dependente Y3 - Performance Financeira distribui-se num intervalo entre -664\% (projetos indicando prejuízo) e 502\% (projetos indicando ganho), conforme Tabela 4.

Tabela 4 - Distribuição de frequência da variável dependente Y3.

\begin{tabular}{|c|c|c|}
\hline PERFORMANCE FINANCEIRA & FREQUÊNCIA & \% \\
\hline Negativo & 367 & 26,46 \\
\hline Zero & 505 & 36,41 \\
\hline Positivo & 515 & 37,13 \\
\hline Total & 1387 & 100,00 \\
\hline
\end{tabular}

Para esta variável, a média é de projetos com Performance Financeira negativa $(-2,68 \%)$, com mediana e moda iguais a zero. Os projetos têm alta variabilidade, com desvio-padrão de $84,40 \%$.

Levando-se em consideração variação de custo, variação de prazo e variação de margem, pode-se dizer que o maior problema na gestão dos projetos aqui analisados se dá em variação de prazo.

\subsection{ANÁLISE DA HIPÓTESE}

Foi conduzida uma análise de correlação, por meio do coeficiente de Pearson, conforme resultados apresentados na Tabela 5. Todas as correlações são consideradas como muito baixas, conforme escala adotada neste trabalho e apresentada na Tabela 6.

Revista de Gestão e Projetos - GeP, São Paulo, v. 3, n. 2, p 178-206, mai./ago. 2012. 
Tabela 5 - Análise de correlação entre as variáveis (coeficiente de Pearson).

\begin{tabular}{|c|c|c|c|c|}
\hline VARIÁVEL & $\mathbf{X 1}$ & $\mathbf{Y 1}$ & $\mathbf{Y 2}$ & $\mathbf{Y 3}$ \\
\hline $\mathbf{X 1}$ & 1 & $-0,043$ & 0,181 & $-0,016$ \\
\hline $\mathbf{Y 1}$ & $-0,043$ & 1 & $-0,231$ & 0,018 \\
\hline $\mathbf{Y 3}$ & 0,181 & $-0,231$ & 1 & 0,09 \\
\hline
\end{tabular}

Tabela 6 - Qualificação de acordo com faixa do índice de correlação.

\begin{tabular}{|c|c|}
\hline QUALIFICAÇÃo & FAIXA DO ÍNDICE DE CORRELAÇ̃̃o \\
\hline Muito baixo & $0,00-0,29$ \\
\hline Baixo & $0,30-0,49$ \\
\hline Moderado & $0,50-0,69$ \\
\hline Alta & $0,70-0,89$ \\
\hline Muito Alta & $0,90-1,00$ \\
\hline
\end{tabular}

No entanto, como observado por Pinto (2002), a questão sobre as formas de avaliação de sucesso sempre foi alvo de polêmica em vários trabalhos, uma vez que sucesso é um fenômeno altamente subjetivo e difícil de ser mensurado objetivamente com uma única variável. Com isso, este trabalho tem, assim como Pinto (2002), três alternativas:

1. Considerar a medida de sucesso/insucesso como uma variável métrica composta a partir dos valores atribuídos para cada dimensão de sucesso. Desta forma, seria criado um índice $\mathrm{R}$ composto pelas $n$ variáveis que medem o sucesso, ou seja, uma variável dependente e $n$ 
variáveis independentes. Assim seria possível aplicar a técnica de análise múltipla de variância. A consequência seria ter que assumir como métrica do trabalho uma variável $R$ que seria o avaliador de sucesso/insucesso;

2. Idem ao item anterior, porém considerando que o índice $\mathrm{R}$ (métrico) fosse dicotômico, por exemplo, classificando o insucesso se $\mathrm{R}<0,5$ e sucesso se $\mathrm{R}>=0,5$;

3. Considerar vários indicadores (métricos ou não-métricos) para avaliar a dimensão de sucesso em projeto. Neste caso, ter-se-ia n variáveis dependentes (métricas) como diferentes medidas de sucesso sendo influenciadas por m variáveis independentes (métricas) como diferentes medidas para a metodologia.

Optou-se, por aprofundar a análise, tratando a variável dependente como sucesso/insucesso do projeto como não-métrica e dicotômica.

Analisou-se cada uma das variáveis dependentes separadamente e considerou-se um projeto no prazo aquele que apresentou variação zero ou positiva em relação ao planejado. Similar análise foi empreendida para custo e performance financeira.

Em seguida, considerou-se se o projeto era um sucesso se as três variáveis dependentes tivessem variação zero ou positiva em relação ao planejado, ou seja, um projeto é um sucesso se ele foi concluído no prazo, dentro do orçamento e com a performance financeira planejada.

Os resultados foram analisados a partir do teste não paramétrico de mediana de Mood (Mood Median Test), que não requer que as populações apresentem distribuição normal, dado que as variáveis não apresentam distribuição normal. A Tabela 7 apresenta a síntese dos resultados.

Tabela 7 - Análise dos relacionamentos entre variáveis.

\begin{tabular}{|c|c|c|c|}
\hline VARIÁVEL X & VARIÁVEL Y & CHI-SQUARE & P-VALUE \\
\hline X1 - Uso de Métodos de Gerenciamento de \\
Projetos & $\begin{array}{c}\text { Y1 - Cumprimento de } \\
\text { Orçmento }\end{array}$ & 2,83 & 0,093 \\
\hline $\begin{array}{c}\text { X1 - Uso de Métodos de Gerenciamento de } \\
\text { Projetos }\end{array}$ & Y2-Cumprimento de Prazo & 21,09 & $0,000^{*}$ \\
\hline
\end{tabular}

Revista de Gestão e Projetos - GeP, São Paulo, v. 3, n. 2, p 178-206, mai./ago. 2012. 


\begin{tabular}{|c|c|c|c|}
\hline $\begin{array}{c}\text { X1 - Uso de Métodos de Gerenciamento de } \\
\text { Projetos }\end{array}$ & $\begin{array}{l}\text { Y3 - Performance } \\
\text { Financeira }\end{array}$ & 1,80 & 0,179 \\
\hline $\begin{array}{c}\text { X1 - Uso de Métodos de Gerenciamento de } \\
\text { Projetos }\end{array}$ & Sucesso & 3,06 & 0,080 \\
\hline
\end{tabular}

*Significativo para $99 \%$ de confiança.

O teste de Mood mostrou relacionamento significativo e positivo entre a variável dependente prazo e a variável independente para um grau de confiança de 99\%. Desta forma, há indícios que quanto maior o grau de implemetação das metodologias de gestão de projetos, maior a chance do projeto cumprir seu prazo planejado. Por outro lado, a variável independente não mostrou impacto significativo com relação às variáveis dependentes custo e performance financeira.

A análise dos projetos considerados sucesso ou fracasso revelou que não existe relacionamento significativo para a variável independente grau de implementação.

\section{CONCLUSÕES}

Este trabalho contribui para sanar uma lacuna na literatura no sentido de se evidenciar o benefício dos investimentos e do uso de métodos de gerenciamento de projetos no sucesso dos projetos, apontada por diversos autores (IKA, 2009; REPISO; SETCHI; SALMERON, 2007; THOMAS; MULLALY, 2008).

Se por um lado, o trabalho reúne evidências de que há benefícios com relação a prazo, por outro, não foi possível constatar os benefícios para questões financeiras, quer com relação a custo, quer com relação à margem.

Esse trabalho traz como implicação para a prática gerencial, evidências dos benefícios de investimentos em tempo no desenvolvimento e uso de métodos de gerenciamento de projetos. No entanto, alerta para o fato de que provavelmente os métodos estejam sendo mais eficazes com relação à gestão dos prazos, do que com relação a questões financeiras. Sugere-se que esses aspectos sejam explorados em uma agenda futura de estudos.

A principal limitação desta pesquisa foi o uso de uma única organização como fonte de informação de projetos, embora extraídas de 10 unidades de negócio de setores industriais distintos. 
No entanto, esta opção trouxe benefícios que dificilmente seriam obtidos, caso se optasse por conduzir análises com diversas organizações. O primeiro deles foi à disponibilidade de informações, obtidas diretamente das bases de dados da organização. Foi possível obter uma amostra grande de projetos (1387), coletados por um extenso período de tempo, com dados detalhados, que em geral são tratados como confidenciais em grande parte das organizações, Além disso, a variável grau de utilização dos métodos de gestão, para sua confiabilidade, precisava ser feita em uma base sistemática e longitudinal. Desta forma, utilizaram-se dados auditados da empresa analisada.

\section{REFERÊNCIAS}

AIPM, AUSTRALIAN INSTITUTE OF PROJECT MANAGEMENT. AIPM Professional Competency Standards for Project Management. Sidney: AIPM, 2008.

APM, ASSOCIATION FOR PROJECT MANAGEMENT. APM Body of Knowledge. Buckinghamshire: APM, 2006.

Archer, N. P.; Ghasemzadeh, F. An integrated framework for project portfolio selection. International Journal of Project Management, v. 17, n. 4, p. 207-216, 1999.

Barber, E. Benchmarking the management of projects: a review of current thinking. International Journal of Project Management, v. 22, p. 301-307, 2004.

Belout, A.; Gauvreau, C. Factors influencing project success: The impact of human resource management. International Journal of Project Management, v. 22, p. 1-11, 2004.

Besner, C.; Hobbs, B. The perceived value and potential contribution of project management practices to project success. Project Management Journal, v. 37, n. 3, p. 37-48, 2006.

Bizan, O. The determinants of success of R\&D projects: Evidence from american-israeli research alliances. Research Policy, v. 32, p. 1619-1640, 2003.

Bryde, D. J. Modelling project management performance. International Journal of Quality \& Reliability Management, v. 20, n. 2, p. 229-254, 2003. 
Buchanan, J. Measuring up. PM Network. Project Management Institute, 2008.

Cooke-Davies, T. J. The "real" success factors on projects. International Journal of Project Management, v.20, p. 185-190, 2002.

Dai, C. X.; Wells, W. G. An exploration of project management office features and their relationship to project performance. International Journal of Project Management, v. 22, p. 523$532,2004$.

Dvir, D; Lipovetsky, S.; Shenhar, A.; Tishler, A. In search of project classification: A non-universal approach to project success factors. Research Policy, v. 27, p. 915-935, 1998.

Dvir, D.; Raz, T.; Shenhar, A. An empirical analysis of the relationship between project planning and project success. International Journal of Project Management, v. 21, p. 89-95, 2003.

Eisenhardt, K. M.; Graebner, M. E. Theory building from cases: opportunities and challenges. Academy of Management Journal, v. 50, no 1, p. 25-32, 2007.

ENAA, ENGINEERING ADVANCEMENT ASSOCIATION OF JAPAN. Model FormInternational Contract for process plant construction. Tokyo: ENAA, 1992.

Gray, R. Organizational climate and project success. International Journal of Project Management, v. 19, p. 103-109, 2001.

Hair, J. F.; Anderson, R. E.; Tatham, R. L.; Black, W. C. Multivariate data analysis. New Jersey: Prentice Hall, 1998.

Hendricks, K.; Singhal, V. R. The long-run stock price performance of firms with effective TQM programs. Management Science, v. 47, n. 3, p. 359-368, 2001.

IBBS, W.; Reginato, J. Quantifying the value of project management. Project Management Institute Inc., Pennsylvania: Newton Square, 2002.

Ibert, O. Projects and firms as discordant complements: Organisational learning in the Munich software ecology. Research Policy, v. 33, p. 1529-1546, 2004.

Ika, L. A. Project success as a topic in project management journals. Project Management Journal, Four Campus Boulevard: Project Management Institute v. 40, n. 4, p. 06-19, 2009.

IPMA, INTERNATIONAL PROJECT MANAGEMENT ASSOCIATION. ICB - IPMA Competency Baseline. Nijkerk: IPMA, 2006.

Revista de Gestão e Projetos - GeP, São Paulo, v. 3, n. 2, p 178-206, mai./ago. 2012. 
Jugdev, K; Muller, R. A retrospective look at our evolving understanding of project success. Project Management Journal, v. 36, n. 4, p. 19-31, 2005.

Katz, R.; Allen, T. J. Project performance and the locus of influence in the R\&D matrix. Academy of Management Journal, v. 28, 1985.

Kendra, K.; Taplin, L. Project success: A cultural framework. Project Management Journal, v. 35, n. 1, p. 30-45, 2004.

Kerzner, H. Project management - A systems approach to planning, scheduling, and controlling. New York: John Wiley \& Sons, 2001.

Kessler, H.; Winkellhofer, G. Projekt-management: Leitfaden zur steuerung und fuhrung von projekten. Heidelberg: Springer, 2002.

Larson, E; Gobeli, D. Significance of project management structure on development success. IEEE Transactions on Engineering Management, v. 36, n. 2, p. 119-125, 1989.

Ling, F. Y. Y. How project managers can better control the performance of design-build projects. International Journal of Project Management, v. 22, p. 477-488, 2004.

Lipovetsky, S.; Tishler, A.; Dvir, D.; Shenhar, A. The relative importance of project success dimensions. R\&D Management, v. 27, n. 2, p. 97-106, 2005.

Marconi, M.; Lakatos, E. Fundamentos de metodologia científica. São Paulo: Atlas, 2003.

Markowitz, H. M. Foundations of portfolio theory. Nobel Lecture, 1990.

OGC, OFFICE OF GOVERNMENT COMMERCE. PRINCE2 - Projects in Controlled Environments. Londres: OGC, 1996.

Patah, L. A.; Carvalho, M. M. Quantifying the value of project management: The actual situation in the it market in Brazil. Resumos... Ankara: EUROMA, 2007.

Pinto, S. A. O. Gerenciamento de projetos: Análise dos fatores de risco que influenciam o sucesso de projetos de sistemas de informação. Dissertação de Mestrado. Departamento de Administração da Faculdade de Economia, Administração e Contabilidade da Universidade de São Paulo, São Paulo, 2002. 
PMI, PROJECT MANAGEMENT INSTITUTE. A Guide to the project management body of knowledge (PMBoK Guide). Forth Edition. Project Management Institute, Four Campus Boulevard, Newtown Square, 2008.

PMI, PROJECT MANAGEMENT INSTITUTE. PMI today June 2009. Project Management Institute, Four Campus Boulevard, Newtown Square, 2009.

Rad, P. F.; Levin, G. Metrics for project management - Formalized approaches. Management Concepts. Vienna, VA, 2006.

Raz, T.; Shenhar, A. J.; Dvir, D. Risk management, project success, and techonological uncertainty. R\&D Management, v. 32, n. 2, p. 101-109, 2002.

Repiso, L.; Setchi, R.; Salmeron, J. Modelling IT projects success: Emerging methodologies reviewed. Technovation, Article in Press, 2007.

Silveira, G. A. Fatores contribuintes para a maturidade em gerenciamento de projetos: Um estudo em empresas brasileiras. Tese de Doutorado. Departamento de Administração da Faculdade de Economia, Administração e Contabilidade da Universidade de São Paulo, São Paulo, 2008.

Söderlund, J. Building theories of project management: Past research, questions for the future. International Journal of Project Management, v. 22, p. 183-191, 2004.

Thamhain, H. J. Linkages of project environment to performance: Lessons for team leadership. International Journal of Project Management, v. 22, p. 533-544, 2004.

THE STANDISH GROUP INTERNATIONAL. Chaos Summary 2009. Disponível em: http://www.standishgroup.com/. Acesso em: 28 dez. 2009.

Thomas, J., Delisle, C. L.; Jugdev, K. Selling project management to senior executives. Project Management Institute Inc., Newtown Square, 2002.

Thomas, J.; Mullaly, M. Researching the value of project management. PMI. Pennsylvania: Newtown Square, 2008.

White, D.; Fortune, J. Current practice in project management - An empirical study. International Journal of Project Management, v. 20, p. 1-11, 2002. 


\section{Anexo 1 - Informações do Projeto}

\begin{tabular}{|c|c|}
\hline TIPO & DESCRIÇÃO \\
\hline Nome do projeto & $\begin{array}{l}\text { Referência segundo a qual o projeto é conhecido dentro da organização. } \\
\text { Esta informação foi alterada por um valor numérico sequencial por } \\
\text { questões de privacidade das informações dos projetos e dos clientes dos } \\
\text { mesmos para a organização }\end{array}$ \\
\hline Período de realização do projeto & $\begin{array}{l}\text { Medido em ano e trimestre de início de realização do projeto. A } \\
\text { informação do trimestre de realização do projeto é apresentada da } \\
\text { seguinte forma: } \\
\text { Q1: } 1^{\circ} \text { trimestre; período que compreende os meses de outubro, } \\
\text { novembro e dezembro do ano anterior ao citado na planilha, de } \\
\text { acordo com o calendário fiscal da empresa, que se inicia em } \\
\text { outubro de um ano e termina em setembro do ano seguinte } \\
\text { Q2: } 2^{\circ} \text { trimestre; período que engloba os meses de janeiro, } \\
\text { fevereiro e março } \\
\text { Q3: } 3^{\circ} \text { trimestre; composto pelos meses de abril, maio e junho } \\
\text { Q4: } 4^{\circ} \text { trimestre; compreendido pelos meses de julho, agosto e } \\
\text { setembro }\end{array}$ \\
\hline Grau de implementação & $\begin{array}{l}\text { Grau de implementação de métodos na divisão que conduziu o projeto } \\
\text { em valor percentual }\end{array}$ \\
\hline $\begin{array}{l}\text { Investimento em treinamento e } \\
\text { capacitação }\end{array}$ & $\begin{array}{l}\text { Valor financeiro em reais do investimento realizado em treinamento e } \\
\text { capacitação de gestores de projetos na divisão que conduziu o projeto }\end{array}$ \\
\hline $\begin{array}{l}\text { Número de gerentes de projetos } \\
\text { certificados como PMP }\end{array}$ & $\begin{array}{l}\text { Quantidade de profissionais certificados PMP pelo PMI na divisão, não } \\
\text { necessariamente na condução do projeto analisado em específico }\end{array}$ \\
\hline $\begin{array}{l}\text { Variação relativa do custo do } \\
\text { projeto }\end{array}$ & $\begin{array}{l}\text { Medida através da variação relativa do aumento de orçamento, calculada } \\
\text { pela diferença em valor monetário do orçamento original previsto pelo } \\
\text { projeto em relação ao orçamento final do projeto, dividida pelo } \\
\text { orçamento inicial }\end{array}$ \\
\hline $\begin{array}{c}\text { Variação relativa do prazo do } \\
\text { projeto }\end{array}$ & $\begin{array}{l}\text { Medida por meio da variação relativa do aumento de prazo do projeto, } \\
\text { calculada através da diferença entre o prazo original planejado em dias e } \\
\text { o prazo final do projeto, dividida pelo prazo inicial }\end{array}$ \\
\hline $\begin{array}{c}\text { Variação relativa da margem do } \\
\text { projeto }\end{array}$ & $\begin{array}{l}\text { Medida por meio da variação relativa da margem do projeto, calculada } \\
\text { através da diferença entre a margem final do projeto e a margem prevista } \\
\text { originalmente, dividida pela margem inicial }\end{array}$ \\
\hline
\end{tabular}

Revista de Gestão e Projetos - GeP, São Paulo, v. 3, n. 2, p 178-206, mai./ago. 2012. 


\begin{tabular}{|c|l|}
\hline Segmento de atuação & $\begin{array}{l}\text { Mercado onde o projeto foi aplicado. Apresentado em componentes, } \\
\text { energia, indústria, manutenção, medicina, óleo e gás, segurança, } \\
\text { tecnologia da informação, telecomunicações e transportes }\end{array}$ \\
\hline País de condução do projeto & País onde o projeto foi conduzido, Argentina, Brasil ou Chile \\
\hline
\end{tabular}

Data do recebimento do artigo: 31/05/2012

Data do aceite de publicação: 23/07/2012

Revista de Gestão e Projetos - GeP, São Paulo, v. 3, n. 2, p 178-206, mai./ago. 2012. 